\title{
Is underwater endoscopic mucosal resection of colon polyps superior to conventional techniques? A network analysis of endoscopic mucosal resection and submucosal dissection
}

\section{(ㄷ)(i) $\odot$}

\section{Authors}

Darren Jun Hao Tan ${ }^{1}$, Cheng Han Ng${ }^{1}$, Xiong Chang Lim¹, Wen Hui Lim¹, Linus Zhen Han Yuen ${ }^{1}$, Jin Hean Koh', Kameswara Rishi Yeshayahu Nistala', Khek-Yu Ho ${ }^{1,2}$, Choon Seng Chong*, ${ }^{*}$, Mark D. Muthiah*,1,2

Institutions

1 Yong Loo Lin School of Medicine, National University of Singapore, Singapore, Singapore

2 Division of Gastroenterology and Hepatology, Department of Medicine, National University Hospital, Singapore, Singapore

3 Division of Colorectal Surgery, Department of Surgery, National University Hospital, Singapore, Singapore

submitted 21.3.2021

accepted after revision 11.8 .2021

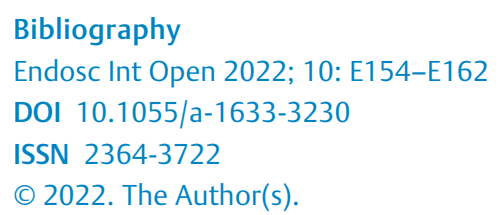

This is an open access article published by Thieme under the terms of the Creative Commons Attribution-NonDerivative-NonCommercial License, permitting copying and reproduction so long as the original work is given appropriate credit. Contents may not be used for commercial purposes, or adapted, remixed, transformed or built upon. (https://creativecommons.org/licenses/by-nc-nd/4.0/)

Georg Thieme Verlag KG, Rüdigerstraße 14,

70469 Stuttgart, Germany

Corresponding author

Dr. Mark Muthiah, MBBS (S'pore), MRCP (UK), MMED (S'pore), Consultant Gastroenterologist and Hepatologist, Division of Gastroenterology and Hepatology, Tower Block Level 10, $1 \mathrm{E}$ Kent Ridge Road, 119228, Singapore

Phone: +65 67724354

mdcmdm@nus.edu.sg

Supplementary material is available under https://doi.org/10.1055/a-1633-3230

\section{ABSTRACT}

Background and study aims Evidence from recent trials comparing conventional endoscopic mucosal resection (EMR) to underwater EMR (UEMR) have matured. However, studies comparing UEMR to endoscopic submucosal dissection (ESD) are lacking. Hence, we sought to conduct a comprehensive network meta-analysis to compare the efficacy of UEMR, ESD, and EMR.

Methods Embase and Medline databases were searched from inception to December 2020 for articles comparing UEMR with EMR and ESD. Outcomes of interest included rates of en bloc and complete polyp resection, risk of perforation and bleeding, and local recurrence. A network meta-analysis comparing all three approaches was conducted. In addition, a conventional comparative meta-analysis comparing UEMR to EMR was performed. Analysis was stratified according to polyp sizes $(<10 \mathrm{~mm}, \geq 10 \mathrm{~mm}$, and $\geq 20 \mathrm{~mm}$ ).

Results Twenty-two articles were included in this study. For polyps $\geq 10 \mathrm{~mm}$, UEMR was inferior to ESD in achieving en bloc resection $(P=0.02)$. However, UEMR had shorter operating time for polyps $\geq 10 \mathrm{~mm}(P<0.001)$, and $\geq 20 \mathrm{~mm}$ $(P=0.019)$ with reduced perforation risk for polyps $\geq 10 \mathrm{~mm}$ $(P=0.05)$ compared to ESD. In addition, en bloc resection rates were similar between UEMR and EMR, although UEMR had reduced recurrence for polyps $\geq 10 \mathrm{~mm}$ ( $P=$ $0.013)$ and $\geq 20 \mathrm{~mm}(P=0.014)$. UEMR also had shorter mean operating than EMR for polyps $\geq 10 \mathrm{~mm}(P<0.001)$ and $\geq 20 \mathrm{~mm}(P<0.001)$. Risk of bleeding and perforation with UEMR and EMR were similar for polyp of all sizes.

Conclusions UEMR has demonstrated technical and oncological outcomes comparable to ESD and EMR, along with a desirable safety profile. UEMR appears to be a safe and effective alternative to conventional methods for resection of polyps $\geq 10 \mathrm{~mm}$.

\footnotetext{
* These authors contributed equally
} 


\section{Introduction}

Colorectal cancer is the second most deadly cancer worldwide, accounting for $10 \%$ of deaths annually [1]. Current prevention strategies entail endoscopic resection of precancerous lesions [2] and recent advancements in endoscopic techniques have improved the rate of en bloc resection, amounting to decreased recurrence rates over the last decade. In particular, the use of underwater endoscopic mucosal resection (UEMR) has gained notability for its perceived benefits [3]. UEMR was first conceived by Binmoeller et al in 2012 and has since been replicated in many centers [4]. The procedure involves insufflation of the lumen with water prior to polyp removal, which has been perceived to allow for easier resection of polyps and reduced risk of thermal injury. Conventionally, resection of polyps has been done via endoscopic mucosal resection (EMR) and endoscopic submucosal dissection (ESD), with evidence from meta-analysis favoring the later in larger lesions. The European Society of Gastrointestinal Endoscopy (ESGE) consensus recommended the use of ESD in lesions $>20 \mathrm{~mm}$ [5]; however, the steep learning curve has limited its application in the West [6]. While evidence from randomized controlled trials comparing UEMR and EMR has matured [7,8], the potential to outperform ESD has yet to be established.

At present, there have been a number of meta-analyses focusing on single-arm outcomes of UEMR and its relative efficacy compared to EMR. However, many of these studies were conducted in fixed-effect models, which have since been subjected to skepticism due to mounting evidence for use of random effects models [9]. In addition, the utility of UEMR and EMR in lesions $<10 \mathrm{~mm}$ has yet to be evaluated. Thus, we performed: (1) a conventional pairwise meta-analysis to compare between UEMR and EMR with random effects models and subgroup analysis for different polyp sizes; and (2) network analysis to compare the efficacy of UEMR vs ESD and UEMR vs EMR.

\section{Methods}

\section{Search strategy}

This review was synthesized according to PRISMA guidelines. A search was conducted on Medline and Embase for articles comparing UEMR with conventional techniques (ESD, EMR) on December 2, 2020. The search strategy used was "underwater adj3 (endoscop* mucosal resect*) or UEMR" in titles and abstracts. References were downloaded into Endnote X9 and duplicates were removed. We previously conducted a meta-analysis comparing ESD and EMR on October 4, 2020 [10] and the data were used to supplement the network meta-analysis.

\section{Inclusion criteria and data extraction}

Two authors (DJHT, CHN) screened references for suitability based on abstracts. We included randomized controlled trials (RCTs) and retrospective and prospective cohort studies that compared UEMR with conventional techniques. Only original articles were included and editorials, commentaries and reviews were excluded. Two authors in a blinded pair (DJHT, CHN) extracted relevant raw data into a structured proforma.
The main outcomes evaluated were rates of en bloc resection, complete resection, perforation, bleeding, operation time, and recurrence. En bloc resection was defined as endoscopically confirmed removal of a polyp in a single piece, while complete resection was defined either with histologically confirmed negative margins or absence of macroscopically visible polyp fragments. Bleeding events were analyzed as a summative of intraprocedural and delayed bleeding. As with previous reviews, recurrence rate was extracted from the last recorded follow-up $[3,11-16]$. Unreported means and standard deviations were converted using pre-existing formulas by Wan et al [17].

\section{Statistical analysis}

First, a conventional pairwise meta-analysis was conducted using the Dersimonian and Laird model. Dichotomous variables were analyzed in risk ratios (RR), and continuous variables in weighted mean difference (WMD). Fixed effect models have many assumptions beyond statistical significance in heterogenicity and many have advocated for use of random effects in meta-analysis [9]. Thus, all analysis was conducted with random effects model regardless of heterogenicity $\left(\mathrm{Tau}^{2}, \mathrm{I}^{2}\right.$, and Cochran Q test). For outcomes where sufficient data was available, prediction intervals were also calculated [18]. Subgroup analysis was considered according to lesion size $(<10 \mathrm{~mm}$, $\geq 10 \mathrm{~mm}$ and $\geq 20 \mathrm{~mm}$ ).

In the network meta-analysis, a frequentist approach as described by lan White [19] was used in the estimation of effect size for indirect comparisons. Only articles with polyps $\geq 10 \mathrm{~mm}$ were considered, with sensitivity analysis for $\geq 20 \mathrm{~mm}$. Recurrence was not included in the network analysis due to significantly different follow-up times between EMR vs ESD and EMR vs UEMR (4.9 vs 22.0 months). The network analysis was done in the natural log of RR and the results were exponentiated for conversion back to RR. Inconsistency testing, which quantifies that results are sufficiently similar for an indirect comparison to be drawn, was done through both local and global approaches using Wald's test and node splitting [19]. Publication bias was evaluated through visual inspection of funnel plots. All analysis was conducted in STATA 16.1 , or $\mathrm{R}$ Studio (version 4.0.3) using the meta package where appropriate. Statistical significance was considered at $\mathrm{P}<0.05$ for all outcomes.

\section{Quality assessment and risk of bias}

RCTs were assessed by the Cochrane Risk-of-Bias 2.0 tool for appropriateness of randomization process, deviation from intended outcomes, missing data, measures of outcomes, and selection of reporting [20]. Non-randomized studies were analyzed with the Newcastle-Ottawa Scale (NOS) [21], which is designed to assess such studies in three domains: (1) selection of the study groups; (2) comparability of the groups; and (3) outcome of interest for cohort studies. Studies with NOS scores of 7-9, 4-6, and $<3$ were interpreted as having low, moderate, and high risk of bias respectively. 


\section{Results}

\section{Summary of included articles}

A total of 194 articles were included in the initial search, with 145 remaining after duplicate removal ( $>$ Fig. 1 ). Thirteen articles from a meta-analysis we previously conducted comparing ESD and EMR were manually added [10]. After screening of abstracts, 22 articles were included in both groups of analysis [7, 8, 22-41]. The network analysis comprised 13 articles for ESD and EMR [29-41] and eight studies comparing UEMR with EMR $[7,8,22-26,28]$. Seven studies were conducted in Japan [7, 29$32,34,37]$, four in the United States [8, 23, 25, 26], three in Korea [33, 35, 41], and one each in China [27], Czech Republic [40], France [39], Italy [36], Spain [22], Taiwan [28], and the United Kingdom [38]. One included study [24] was a multicenter trial involving centers in Italy and the United States. There were four RCTs, with the remaining being cohort studies. Supplementary material 1 summarizes the characteristics of included studies, including the risk of bias and quality assessment for RCTs and non-randomized studies respectively. In brief, four of the nonrandomized studies included in the analysis had a NOS score $>7$, representing a low risk of bias, while the remaining non-randomized study had a NOS score of 6, corresponding to a moderate risk of bias. In addition, all the included RCTs had low concern for risk of bias as assessed via the Cochrane Risk-of-Bias 2.0 tool, with the exception of one study done by Hamerski et al [24], which demonstrated possible sources of bias from incomplete reporting of data.

\section{Comparative (pairwise) meta-analysis}

A total of 1,551 polyps were included for pairwise analysis of UEMR and EMR. A total of 733 polyps were resected via UEMR while 818 polyps were removed using conventional EMR. A summary of the results is presented in > Table 1 and a summary of the prediction intervals in included in Supplementary material 3.

\section{Polyps $<10 \mathrm{~mm}$}

Pooled analysis of 486 polyps $<10 \mathrm{~mm}$ revealed no statistically significant differences between UEMR and EMR in achieving en bloc (RR: 0.992, Cl: 0.740-1.330, $P=0.955$, > Fig. 2) and complete resection (RR: $0.999, \mathrm{Cl}: 0.768-1.299, P=0.973$ ). Interestingly, pooled analysis demonstrated that UEMR had longer operating time than EMR (WMD: 0.162 mins, Cl: 0.102-0.223 minutes, $P<0.001)$. There was no statistically significant difference in perforation and bleeding for polyps $<10 \mathrm{~mm}$.

\section{Polyps $\geq 10 \mathrm{~mm}$}

From pooled analysis of 1,170 polyps, there was no statistically significant difference between UEMR and EMR in terms of en bloc resection (RR: $1.197, \mathrm{Cl}: 0.922-554, P=0.137$, prediction interval: 0.699-2.050, > Fig.2) and complete resection (RR: 1.122 , Cl: $0.906-1.389, P=0.185$, prediction interval: $0.505-$ 2.492). Pooled analysis of 1,409 polyps also demonstrated that UEMR was associated with shorter procedure times (WMD: -1.364 mins, $\mathrm{Cl}:-1.754$ to -0.975 minutes, prediction interval -15.458 to $5.627, P<0.001)$. UEMR was also associated

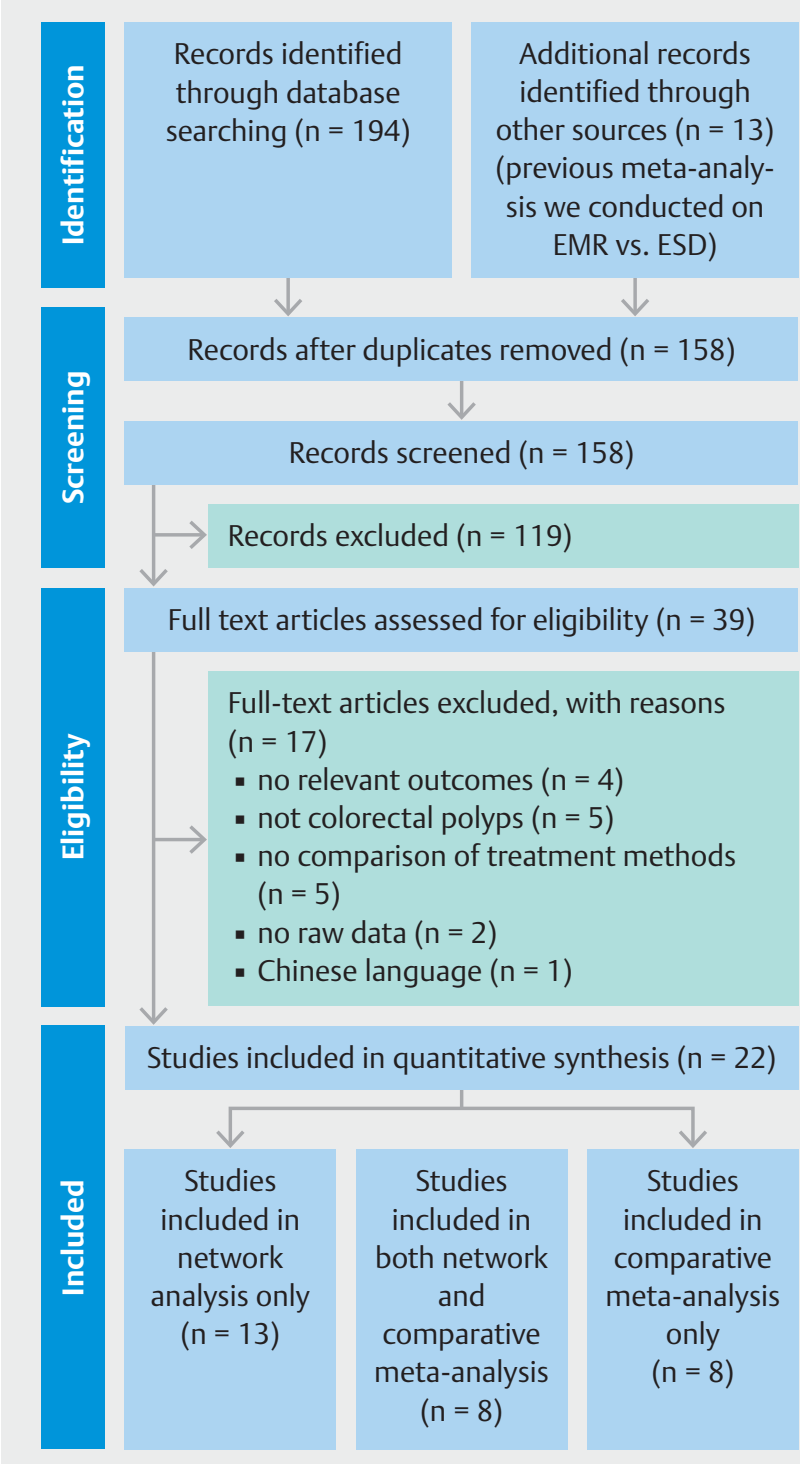

-Fig. 1 PRISMA flow diagram of included articles.

with lower recurrence (RR: $0.517, \mathrm{Cl}: 0.286-0.935, P=0.036$, prediction interval: $0.240-1.116)$. There was no statistically significant difference in perforation and bleeding risk.

\section{Polyps $\geq 20 \mathrm{~mm}$}

From analysis of 479 polyps $\geq 20 \mathrm{~mm}$, the difference in rate of en bloc RR: $1358, \mathrm{Cl}: 0.624-2.955, P=0.299$, prediction interval: $0.214-8.636$, > Fig. 2 ) and complete resection (RR: 0.930, $\mathrm{Cl}: 0.793-1.113, P=0.470)$ did not reach statistical significance. UEMR continued to be associated with shorter operating time (WMD: -2.253 mins, $\mathrm{Cl}:-2.693$ to -1.812 minutes, $P<$ $0.001)$. UEMR was also superior in terms of reduced local recurrence after resection of larger polyps (RR: $0.169, \mathrm{Cl}$ : $0.053-$ $0.542, P=0.003)$. There was no statistically significant difference in perforation and bleeding. 
- Table 1 Summary of pooled analysis comparing UEMR and EMR.

\begin{tabular}{|c|c|c|c|c|c|}
\hline Outcomes & Total studies & Events & Total sample & Effect size $(95 \% \mathrm{Cl})$ & $P$ \\
\hline \multicolumn{6}{|l|}{$\leq 10 \mathrm{~mm}$} \\
\hline - On-piece resection & 2 & 470 & 486 & $0.992(0.740-1.330)$ & 0.955 \\
\hline - Complete resection & 2 & 458 & 486 & $0.999(0.768-1.299)$ & 0.973 \\
\hline - Perforation & 2 & 2 & 486 & $0.955(0.528-1.725)$ & 0.500 \\
\hline - Bleeding & 2 & 5.5 & 486 & $0.367(0.059-2.275)$ & 0.091 \\
\hline - Mean Operation Time & 2 & NA & 486 & 0.162 mins ( 0.102 to 0.223 ) & $<0.001$ \\
\hline \multicolumn{6}{|l|}{$\geq 10 \mathrm{~mm}$} \\
\hline - One-piece resection & 6 & 698 & 1170 & $1.197(0.922-1.554)$ & 0.137 \\
\hline - Complete resection & 4 & 517 & 625 & $1.122(0.906-1.389)$ & 0.185 \\
\hline - Perforation & 7 & 10.5 & 1219 & $1.042(0.504-2.154)$ & 0.894 \\
\hline - Bleeding & 8 & 85.5 & 1409 & $1.004(0.489-2.064)$ & 0.989 \\
\hline - Recurrence & 5 & 111 & 624 & $0.517(0.286-0.935)$ & 0.036 \\
\hline - Mean Operation Time & 5 & NA & 1409 & -1.364 mins $(-1.754$ to -0.975$)$ & $<0.001$ \\
\hline \multicolumn{6}{|l|}{$\geq 20 \mathrm{~mm}$} \\
\hline - One-piece resection & 4 & 186 & 479 & $1.358(0.624-2.955)$ & 0.299 \\
\hline - Complete resection & 1 & 31 & 32 & $0.939(0.793-1.113)$ & 0.470 \\
\hline - Perforation & 1 & 1 & 32 & $1.000(0.021-47.380)$ & 1.00 \\
\hline - Bleeding & 1 & 5 & 32 & $1.500(0.288-7.807)$ & 0.630 \\
\hline - Recurrence & 1 & 15 & 67 & $0.169(0.053-0.542)$ & 0.003 \\
\hline - Mean operation time & 2 & NA & 546 & -2.253 mins $(-2.693$ to -1.812$)$ & $<0.001$ \\
\hline
\end{tabular}

\section{Network meta-analysis}

The network diagram for the comparison of UEMR vs ESD and UEMR is represented in > Fig. 3. Interval plots of the network analysis are represented in $\mathbf{F i g . 4}$. Results of the analysis are summarized in $\mathbf{r}$ Table 2 . For all outcomes, global test of inconsistency and local node splitting did not reveal any significance for inconsistency. No publication bias was observed aside from complete resection and operation time from visual inspection of funnel plots (Supplementary material 4). The summary of GRADE evidence can be found in Supplementary material 5.

Polyps $\geq 10 \mathrm{~mm}$

UEMR was found to be inferior to ESD in achieving en bloc resection (RR: $0.645, \mathrm{Cl}: 0.445-0.934, P=0.020$ ). There was no statistically significant difference in achieving complete resection (RR: $0.786, \mathrm{Cl}$ : $0.533-1.160, P=0.226$ ). UEMR was associated with statistically significantly decreased procedure time compared to ESD (WMD: -69.61 mins, $\mathrm{Cl}$ : -101.63 to -37.59 minutes, $P<0.001)$. UEMR was also associated with reduced risk of perforation (RR: $0.273, \mathrm{Cl}: 0.0731-1.012, P=0.05$ ) but bleeding risk did not significantly differ (RR: $0.89, \mathrm{Cl}: 0.35-$ 2.28, $P=0.807$ )
Comparing UEMR and EMR via network analysis, there was no statistically significant difference for en bloc (RR: $1.200, \mathrm{Cl}$ :0.902-1.595, $P=0.211$ ) and complete resection (RR: 1.132 , $\mathrm{Cl}$ : $0.836-1.533, P=0.423$ ) of polyps $\geq 10 \mathrm{~mm}$. There were no statistically significant differences in perforation (RR: 1.141 $\mathrm{Cl}: 0.336-3.874, P=0.832$ ) and bleeding (RR: $1.049 \mathrm{Cl}: 0.522-$ 2.107, $P=0.894)$ for UEMR and EMR.

\section{Polyps $\geq 20 \mathrm{~mm}$}

When sensitivity analysis was conducted for polyps $\geq 20 \mathrm{~mm}$, there was no statistically significant difference between UEMR and ESD in achieving en bloc (RR: 0.698, Cl: 0.396-1.230, $P=$ 0.214 ) and complete resection (RR: $0.612, \mathrm{Cl}: 0.245-1.526, P$ $=0.292$ ). Operating time was significantly shortened in UEMR (WMD: -52.56 mins, $\mathrm{Cl}$ : -96.38 to -8.73 mins, $P<0.019)$. There was no statistically significant difference for perforation (RR:0.201, Cl:0.004-9.993, $P=0.421$ ) and bleeding (RR: 1.606 $\mathrm{Cl}$ :0.280-9.207, $P=0.595)$ between UEMR and ESD.

Comparing UEMR to EMR, there was no statistically significant difference for en bloc (RR: 1.349, Cl: 0.840-2.168, $P=$ 0.215 ) and complete resection (RR: $0.941, \mathrm{Cl}: 0.417-2.123, P$ $=0.883)$ of polyps $\geq 20 \mathrm{~mm}$. UEMR also did not have statistically 


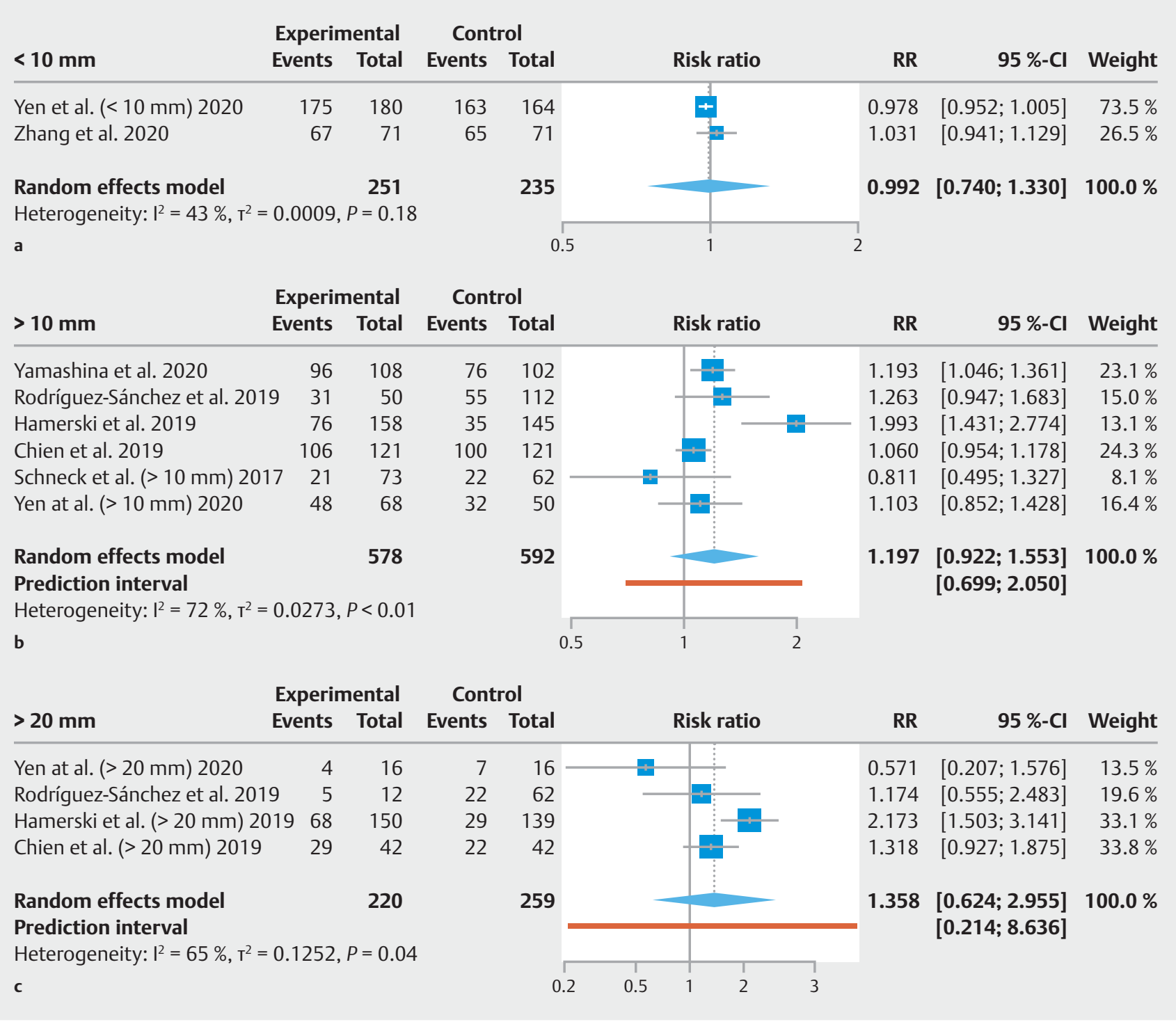

- Fig. 2 Forest plot of UEMR vs EMR for en bloc resection stratified by polyp size.

significant benefit in terms of perforation (RR: $1.000 \mathrm{Cl}: 0.021-$ 47.510, $P=1.00$ ) and bleeding (RR: $1.5 \mathrm{Cl}: 0.288-7.805, P=$ $0.63)$.

\section{Discussion}

Utilizing both conventional pairwise meta-analysis and network meta-analysis, we demonstrated that UEMR might be superior to EMR and ESD for resection of colon polyps $\geq 10 \mathrm{~mm}$, but offered no advantage for polyps $<10 \mathrm{~mm}$. For colon polyps $\geq 10 \mathrm{~mm}$, we demonstrated a significant reduction in procedure time for UEMR compared with both EMR and ESD. UEMR also had a lower recurrence rate than EMR, and a lower risk of perforation as compared to ESD. When comparing UEMR with ESD for polyps $\geq 10 \mathrm{~mm}$, there was a reduced rate of en bloc resection, although rates of complete resection were similar. A further sensitivity analysis for polyps $\geq 20 \mathrm{~mm}$ also demonstrated a significant reduction in procedure time with UEMR compared with both EMR and ESD, and a lower recurrence rate with UEMR as compared to EMR. Only for polyps $<10 \mathrm{~mm}$ did UEMR require a longer procedure time than EMR.

These findings have significant implications for delivery of care. We demonstrated the superiority of UEMR in resection of polyps $\geq 10 \mathrm{~mm}$ compared to EMR, as improved recurrence rates can lead to reduction in colorectal cancer rates. Compared to ESD, the safety of UEMR, coupled with the reduced procedure times is likely to reduce utilization of healthcare resources. Given the steep learning curve for ESD, use of UEMR may also allow more non-tertiary center-based endoscopists to successfully resect larger colonic polyps, reducing the barrier to care.

UEMR was found to a safety profile similar to conventional methods in terms of perforation and bleeding. The process of underwater emersion increases buoyancy of the lesion, resulting in separation from the muscularis layer and decreasing the risk of adverse events including perforation or deep thermal injury $[4,15]$. Also, while the rate of perforation was significant- 


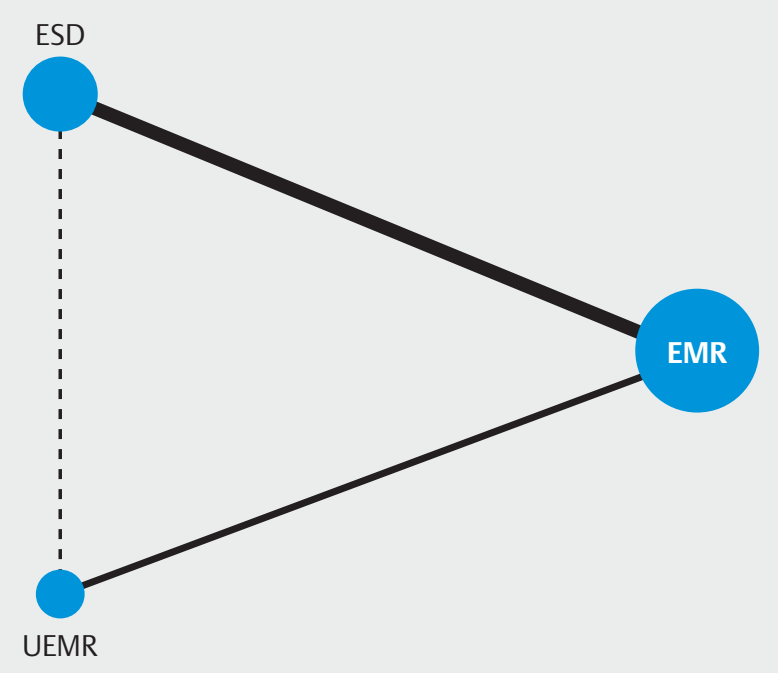

- Fig. 3 Meta-analysis network diagram.

ly lower compared to that of ESD ( $R R=0.273, C l: 0.0731-1.012$, $P=0.05)$, it was non-significant when studies were limited to $\geq 20 \mathrm{~mm}$. However, the analysis should be interpretated with caution as there were limited studies in UEMR with $\geq 20 \mathrm{~mm}$ $(n=2)$.

Several previous meta-analyses have studied the comparative benefits of UEMR (Supplementary material 2). However, we differ from previous articles with respect to two major points. First, our pairwise analysis between UEMR vs EMR is the first to summarize evidence from polyps $<10 \mathrm{~mm}$, and all other analyses were performed in fixed effect models. Next, our network analysis is the first to compare the efficacy between UEMR and ESD, as well as to use current knowledge (ESD vs EMR) to supplement the comparisons from UEMR and EMR. A recent meta-analysis by Choi et al [3] found significant improvements in en bloc resection by UEMR, although these results were conducted in fixed effect models (I2: $38 \%$, Cochrane Q: $P=0.14$ ). Our analysis however found no significant improvement in en bloc resection in both conventional pairwise random effects $P$ $=0.284$ ) and subsequent network analysis $P=0.423$ ). In line with existing literature, ESD was found to be superior in achieving en bloc resection for polyps $\geq 10 \mathrm{~mm}$ [5]. However, sensitivity analysis for polyps $\geq 20 \mathrm{~mm}$ in our network analysis found no significant difference between UEMR and ESD in achieving en bloc resection. This could be the result of the lack of studies for polyps $\geq 20 \mathrm{~mm}$ in the UEMR population that could be utilized in the network analysis. Thus, the lack of statistical power could have given rise to non-significance, rather than a non-inferiority in achieving en bloc resection between UEMR and ESD.

Existing literature has demonstrated reduced local recurrence in UEMR compared to EMR $[14,15]$ and attributed it to higher rates of en bloc resection [42]. In our study, we demonstrated reduced rates of local recurrence with UEMR despite similar rates of en bloc resection compared to EMR We believe there are additional benefits to UEMR that could have contributed to decreased local recurrence. As noted by Binmoeller et al [4], water immersion results in the lesion floating upwards into the lumen due to increased buoyancy of the mucosal and submucosal layers compared to the underlying muscularis, facilitating snaring of the polyp. Water immersion is also postulated to minimize luminal distention and loop formation [43], and

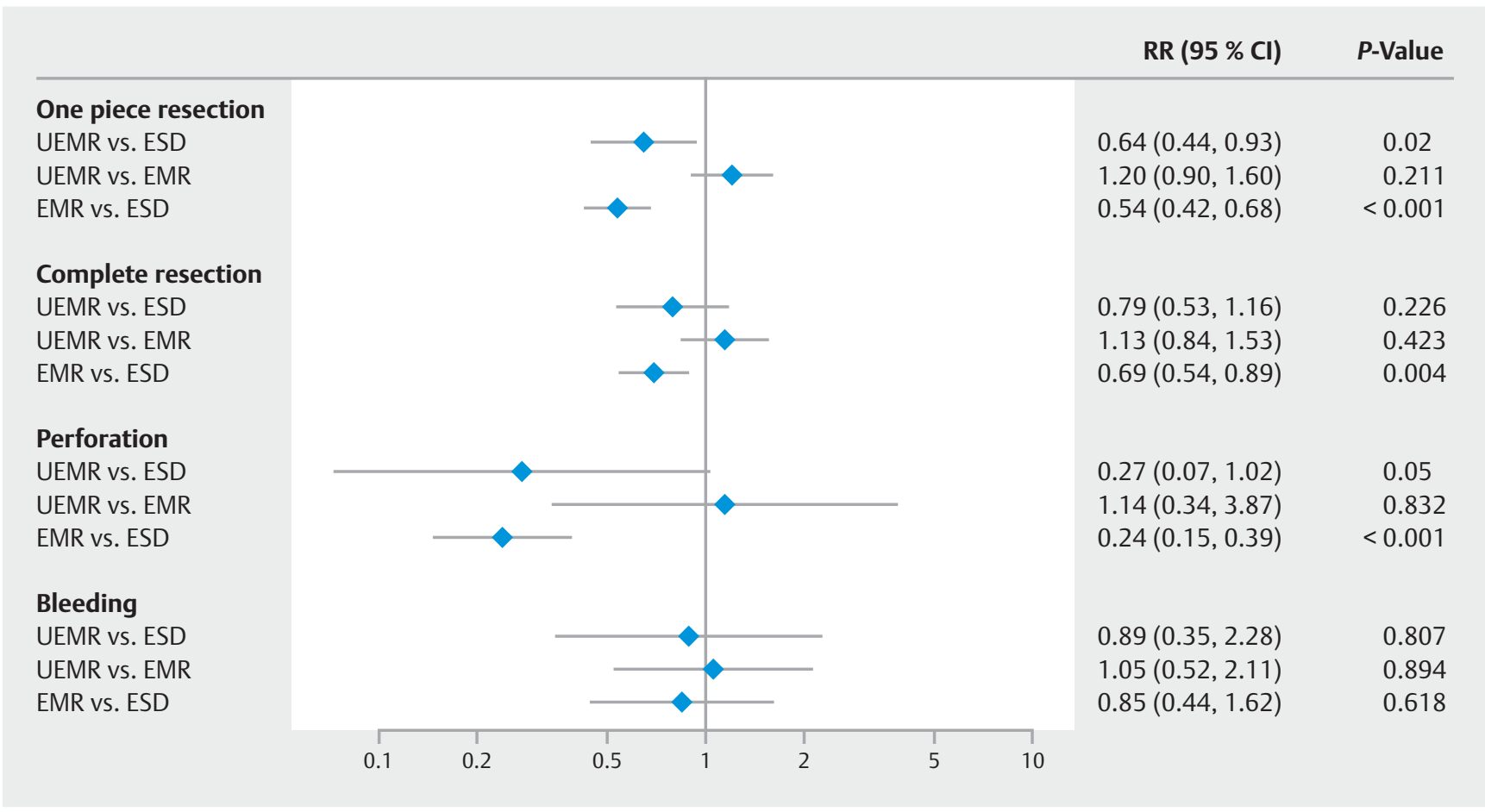

- Fig. 4 Forest plot of network analysis comparing UEMR, EMR, and ESD. 
- Table2 Summary of network analysis comparing UEMR, EMR, and ESD.

\begin{tabular}{|c|c|c|c|c|}
\hline & Polyp size $\geq 10 \mathrm{~mm}$ & & Polyp size $\geq 20 \mathrm{~mm}$ & \\
\hline One-piece resection & Effect size & $P$ value & Effect size & $P$ value \\
\hline UEMR vs ESD & $0.645 \mathrm{Cl}: 0.445-0.934$ & 0.02 & $0.698 \mathrm{Cl}: 0.396-1.230$ & 0.214 \\
\hline UEMR vs EMR & $1.200 \mathrm{Cl}: 0.902-1.595$ & 0.211 & 1.349 Cl: $0.840-2.168$ & 0.215 \\
\hline \multicolumn{5}{|l|}{ Complete resection } \\
\hline - UEMR vs ESD & $0.786 \mathrm{Cl}: 0.533-1.160$ & 0.226 & $0.612 \mathrm{Cl}: 0.245-1.526$ & 0.292 \\
\hline - UEMR vs EMR & $1.132 \mathrm{Cl}: 0.836-1.533$ & 0.423 & $0.941 \mathrm{Cl}: 0.417-2.123$ & 0.883 \\
\hline \multicolumn{5}{|l|}{ Perforation } \\
\hline - UEMR vs ESD & $0.273 \mathrm{Cl}: 0.0731-1.012$ & 0.05 & $0.201 \mathrm{Cl}: 0.004-9.993$ & 0.421 \\
\hline - UEMR vs EMR & $1.141 \mathrm{Cl}: 0.336-3.874$ & 0.832 & $1.000 \mathrm{Cl}: 0.021-47.510$ & 1 \\
\hline \multicolumn{5}{|l|}{ Bleeding } \\
\hline - UEMR vs ESD & $0.889 \mathrm{Cl}: 0.348-2.276$ & 0.807 & $1.606 \mathrm{Cl}: 0.280-9.207$ & 0.595 \\
\hline - UEMR vs EMR & $1.049 \mathrm{Cl}: 0.522-2.107$ & 0.894 & $1.5 \mathrm{Cl}: 0.288-7.805$ & 0.63 \\
\hline \multicolumn{5}{|l|}{ Operation Time } \\
\hline - UEMR vs ESD & $-69.61 \mathrm{Cl}:-101.63$ to -37.59 & $<0.001$ & $-52.56 \mathrm{Cl}:-96.38$ to -8.73 & 0.019 \\
\hline - UEMR vs EMR & $-2.87 \mathrm{Cl}:-26.71$ to 20.98 & 0.652 & $6.30 \mathrm{Cl}:-28.69$ to 41.29 & 0.515 \\
\hline
\end{tabular}

provides a natural optical magnification effect [42], enabling improved visualization and inspection of residual neoplasia. This is of particular importance for larger polyps as EMR has traditionally been associated with low rates of en bloc resection for polyps $\geq 20 \mathrm{~mm}$ and correspondingly high rates of recurrent neoplasia [44].

Due to the significantly different follow-up times for UEMR and ESD (4.9 vs 22.0 months), we did not analyze recurrence rates in the network meta-analysis. However, a previous single-arm analysis estimated a recurrence rate of $9 \%$ in UMER [45], compared to $1 \%$ in ESD [46]. Due to advantages in achieving en bloc resection, ESD has the major advantage of decreased recurrence rates compared to other techniques, and UEMR is unlikely to outperform ESD in terms of local recurrence.

\section{Strengths and limitations}

To our knowledge, this is the first study that compares UEMR and ESD via a network meta-analysis. This allows for evaluation of outcomes despite a paucity of studies comparing the two treatments. A large sample size of $>1000$ polyps was also involved. Finally, we considered outcomes in smaller polyps $<10$ $\mathrm{mm}$ in the comparison of UEMR vs EMR in order to address heterogeneity possibly arising from differing polyp sizes, which was not included in previous studies. Notwithstanding the rigorous search strategy and statistical methodology, we are limited by the lack of reporting of follow-up duration in several included studies which can affect long term outcomes (e.g. recurrence). The procedures described are also operator-dependent and we were unable to quantify the skills of individual endoscopists. In addition, due to the limited number of mature clinical trials, we included data from cohort studies in our network analysis, and confounding factors from the selection of patients could have led to biased estimates. However, consistency was not violated throughout the analysis, as shown via node splitting and Wald's test, demonstrating that the results were sufficiently statistically homogenous for an indirect comparison to be made. Finally, we could not account for heterogeneity due to colonoscope models or snare types used in different centers due to a scarcity in reporting. The definition of complete resection also varied with some articles defining it based on visual inspection.

\section{Conclusions}

There was no statistically significant benefit of conventional methods in achieving technical outcomes, including one-piece and complete resection, in comparison to UEMR. Combined with the significantly reduced procedural time for UEMR, decreased concern for perforation, and shorter training period to achieve proficiency, this study provides evidence that UEMR is a viable and safe alternative for resection of colonic polyps.

\section{Competing interests}

The authors declare that they have no conflict of interest. 


\section{References}

[1] Bray F, Ferlay J, Soerjomataram I et al. Global cancer statistics 2018 GLOBOCAN estimates of incidence and mortality worldwide for 36 cancers in 185 countries. CA Cancer J Clin 2018; 68: 394-424

[2] Shaukat A, Kaltenbach T, Dominitz JA et al. Endoscopic recognition and management strategies for malignant colorectal polyps: recommendations of the US Multi-Society Task Force on Colorectal Cancer. Gastroenterology 2020; 159: 1916-1934.e1912

[3] Choi AY, Moosvi ZM, Shah S et al. Underwater versus conventional endoscopic mucosal resection for colorectal polyps: a systematic review and meta-analysis. Gastrointest Endosc 2020: doi:10.1016/j. gie.2020.10.009

[4] Binmoeller KF, Weilert F, Shah J et al. "Underwater" EMR without submucosal injection for large sessile colorectal polyps (with video). Gastrointest Endosc 2012; 75: 1086-1091

[5] Pimentel-Nunes P, Dinis-Ribeiro M, Ponchon T et al. Endoscopic submucosal dissection: European Society of Gastrointestinal Endoscopy (ESGE) Guideline. Endoscopy 2015; 47: 829-854

[6] Sagawa T, Kakizaki S, lizuka H et al. Analysis of colonoscopic perforations at a local clinic and a tertiary hospital. World J Gastroenterol 2012; 18: 4898-4904

[7] Yamashina T, Uedo N, Akasaka T et al. Comparison of underwater vs conventional endoscopic mucosal resection of intermediate-size colorectal polyps. Gastroenterology157: 451-461.e452

[8] Yen AW, Leung JW, Wilson MD et al. Underwater versus conventional endoscopic resection of nondiminutive nonpedunculated colorectal lesions: a prospective randomized controlled trial (with video). Gastrointest Endosc 2020; 91: 643-654.e642

[9] Tufanaru C, Munn Z, Stephenson M et al. Fixed or random effects meta-analysis? Common methodological issues in systematic reviews of effectiveness. Int J Evid Based Healthc 2015; 13: 196-207

[10] Lim XC, Nistala KRY, Ng CH et al. Endoscopic submucosal dissection vs endoscopic mucosal resection for colorectal polyps: A meta-analysis and meta-regression with single arm analysis. World J Gastroenterol 2021; 27: 3925-3939

[11] Arezzo A, Passera R, Marchese N et al. Systematic review and meta-analysis of endoscopic submucosal dissection vs endoscopic mucosal resection for colorectal lesions. United European Gastroenterol ] 2016; 4: 18-29

[12] De Ceglie A, Hassan C, Mangiavillano B et al. Endoscopic mucosal resection and endoscopic submucosal dissection for colorectal lesions: A systematic review. Crit Rev Oncol Hematol 2016; 104: 138-155

[13] Garg R, Singh A, Mohan BP et al. Underwater versus conventional endoscopic mucosal resection for colorectal lesions: A systematic review and meta-analysis. Endosc Int Open 2020; 8: E1884-E1894

[14] Li P, Ma B, Gong S et al. Underwater endoscopic mucosal resection for colorectal lesions: a meta-analysis. Surgical Endoscopy 2020: doi:10.1007/s00464-020-07745-8

[15] Li DF, Lai MG, Yang MF et al. The efficacy and safety of underwater endoscopic mucosal resection for $\geq 10$-mm colorectal polyps: Systematic review and meta-analysis. Endoscopy 2020: doi:10.1055/a1234-8918

[16] Liu Y, Shi M, Ren J et al. Effectiveness of underwater endoscopic mucosal resection versus conventional endoscopic mucosal resection for 10 to $20 \mathrm{~mm}$ colorectal polyps: A protocol of systematic review and meta-analysis. Medicine 2020; 99: e23041

[17] Wan X, Wang W, Liu J et al. Estimating the sample mean and standard deviation from the sample size, median, range and/or interquartile range. BMC Med Res Methodol 2014; 14: 135

[18] Riley RD, Higgins JPT, Deeks JJ. Interpretation of random effects meta-analyses. BMJ 2011; 342: d549
[19] White IR. Network Meta-analysis. Stata J 2015; 15: 951-985

[20] Sterne JAC, Savović J, Page M] et al. RoB 2: a revised tool for assessing risk of bias in randomised trials. BMJ 2019; 366: 14898

[21] Wells G, Shea B, OConnell DL et al. The Newcastle-Ottawa Scale (NOS) for Assessing the Quality of Nonrandomised Studies in Meta-Analyses. Ottawa Health Research Institute Web site; http://www.ohri.ca/ programs/clinical_epidemiology/oxford.asp (25.12.2020)

[22] Rodríguez-Sánchez J, Uchima-Koecklin H, González-López L et al. Short and long-term outcomes of underwater EMR compared to the traditional procedure in the real clinical practice. Revista Espanola de Enfermedades Digestivas 2019; 111: 543-549

[23] Mouchli M, Walsh C, Reddy S et al. Outcomes of GI polyps resected using underwater endoscopic mucosal resection (UEMR) compared to conventional EMR (CEMR). Gastroenterology 2019; 156: S379-S380

[24] Hamerski C, Samarasena J, Lee DP et al. Underwater versus conventional endoscopic mucosal resection for the treatment of colorectal laterally spreading tumors: Results from an International, Multicenter, randomized controlled trial. Am J Gastroenterol 2019; 114: S75

[25] Schenck RJ, Jahann DA, Patrie JT et al. Underwater endoscopic mucosal resection is associated with fewer recurrences and earlier curative resections compared to conventional endoscopic mucosal resection for large colorectal polyps. Surg Endosc 2017; 31: 4174-4183

[26] Gohil V, Wu Y, Rashid S et al. Underwater endoscopic mucosal resection (UEMR) vs. saline assisted endoscopic mucosal resection (EMR): Does one confer an advantage over the other? Gastrointest Endosc 2015; 81: AB287-AB288

[27] Zhang Z, Xia Y, Cui H et al. Underwater versus conventional endoscopic mucosal resection for small size non-pedunculated colorectal polyps: A randomized controlled trial. BMC Gastroenterol 2020; 20: 311

[28] Chien HC, Uedo N, Hsieh PH. Comparison of underwater and conventional endoscopic mucosal resection for removing sessile colorectal polyps: A propensity-score matched cohort study. Endosc Int Open 2019; 7: E1528-E1536

[29] Tamegai Y, Saito Y, Masaki $N$ et al. Endoscopic submucosal dissection: a safe technique for colorectal tumors. Endoscopy 2007; 39: 418-422

[30] lizuka H, Okamura S, Onozato Y et al. Endoscopic submucosal dissection for colorectal tumors. Gastroenterol Clin Biol 2009; 33: 10041011

[31] Saito Y, Fukuzawa M, Matsuda T et al. Clinical outcome of endoscopic submucosal dissection versus endoscopic mucosal resection of large colorectal tumors as determined by curative resection. Surg Endosc 2010; 24: 343-352

[32] Tajika M, Niwa Y, Bhatia V et al. Comparison of endoscopic submucosal dissection and endoscopic mucosal resection for large colorectal tumors. Eur J Gastroenterol Hepatol 2011; 23: 1042-1049

[33] Lee E], Lee JB, Lee SH et al. Endoscopic treatment of large colorectal tumors: comparison of endoscopic mucosal resection, endoscopic mucosal resection-precutting, and endoscopic submucosal dissection. Surg Endosc 2012; 26: 2220-2230

[34] Terasaki M, Tanaka S, Oka S et al. Clinical outcomes of endoscopic submucosal dissection and endoscopic mucosal resection for laterally spreading tumors larger than $20 \mathrm{~mm}$. J Gastroenterol Hepatol 2012; 27: 734-740

[35] Kim Y], Kim ES, Cho KB et al. Comparison of clinical outcomes among different endoscopic resection methods for treating colorectal neoplasia. Dig Dis Sci 2013; 58: 1727-1736

[36] Acquistapace F, Maternini F, Snider L et al. Endoscopic treatment of superficial colorectal neoplasms. Retrospective analysis of a single center technique and results. G Chir 2015; 36: 247-250

[37] Sato T, Fukuzawa M, Gotoda T et al. Comparison of clinical outcomes between colorectal EMR and ESD. J Tokyo Med University 2016; 74: 154-162 
[38] Emmanuel A, Gulati S, Burt M et al. Combining eastern and western practices for safe and effective endoscopic resection of large complex colorectal lesions. Europ J Gastroenterol Hepatol 2018; 30: 506-513

[39] Soliman H, Brieau B, Guillaumot MA et al. Invasive pit pattern, macronodule and depression are predictive factors of submucosal invasion in colorectal laterally spreading tumours from a Western population. United Europ Gastroenterol J 2018; 6: 1569-1577

[40] Urban O, Falt P, Fojtik P et al. Comparison of endoscopic mucosal resection and endoscopic submucosal dissection in the treatment of flat neoplastic lesions in the rectum. Gastroenterol Hepatol 2018; 72 : 193-198

[41] Jung JS, Hong JY, Oh HH et al. Clinical outcomes of endoscopic resection for colorectal laterally spreading tumors with advanced histology. Surg Endosc 2019; 33: 2562-2571
[42] Belderbos TD, Leenders M, Moons LM et al. Local recurrence after endoscopic mucosal resection of nonpedunculated colorectal lesions: systematic review and meta-analysis. Endoscopy 2014; 46: 388-402

[43] Leung FW, Leung JW, Siao-Salera RM et al. The water method significantly enhances proximal diminutive adenoma detection rate in unsedated patients. J Interv Gastroenterol 2011; 1: 8-13

[44] Hotta K, Fujii T, Saito Y et al. Local recurrence after endoscopic resection of colorectal tumors. Int J Colorectal Dis 2009; 24: 225-230

[45] Spadaccini M, Fuccio L, Lamonaca L et al. Underwater EMR for colorectal lesions: a systematic review with meta-analysis (with video). Gastrointest Endosc 2019; 89: 1109-1116.e1104

[46] Akintoye E, Kumar N, Aihara $\mathrm{H}$ et al. Colorectal endoscopic submucosal dissection: a systematic review and meta-analysis. Endosc Int Open 2016; 4: E1030-E1044 